\title{
Together towards an early global detection of type 2 diabetes using a simplified metabolomics' test
}

\author{
Mina T Kelleni* \\ Department of Pharmacology, Faculty of Medicine, Minia University, Egypt
}

\begin{abstract}
In the era of metabolomics, especially in the past few years; many novel type 2 diabetes molecular biomarkers have been identified and proved to be very beneficial in early detection of the disease that is considered one of the most globally distributed and spreading diseases. Some of these biomarker metabolites seem to be pioneers for developing new global metabolomics' diagnostic as well as prognostic test(s) that hopefully would soon become crucial for early detection and thus early prevention of diabetes complication.
\end{abstract}

\begin{abstract}
Abbreviations: IFG: Impaired Fasting Glucose; IGT: Impaired Glucose Tolerance; T2D: Type 2 diabetes

Diabetes represents one of the most important global health problemsand approximately $90 \%$ of diabetics have type 2 diabetes (T2D) which is called the burden of the 21 st century and is growing with an epidemic rate $[1,2]$. T2D is caused by a complex set of interactions between genetic and environmental factors as well as involvement of multiple organ systems dysfunction; a complexity that presents challenges for a full understanding of the molecular pathways that contribute to the development of this major disease. Progress in this area has been aided by the advent of technologies for comprehensive metabolic analysis; "metabolomics" [3]. Metabolomics is an analytical approach that aims to detect and quantify small metabolites. Recently, there has been an increased interest in the application of metabolomics to the identification of disease biomarkers and to unravel the intricate relationships between metabolism and progression to diabetes as well as monitoring of the disease [4]. Obviously, identification of effective screening markers is critical for early treatment and intervention that can delay and/or prevent complications associated with this chronic disease [1].
\end{abstract}

In 2012, Wang-Sattler and colleagues identified after quantifying 140 metabolites for 4297 fasting serum samples in the populationbased Cooperative Health Research in the Region of Augsburg (KORA) cohort, three metabolites; (glycine, lysophosphatidylcholine (C18:2 LPC) and acetylcarnitine) that had significantly altered levels in impaired glucose tolerance (IGT) individuals as compared to those with normal glucose tolerance. They've also confirmed that lower levels of glycine and LPC were found to be predictors not only for IGT but also for T2D, and these three metabolites, among others, were independently confirmed in the European Prospective Investigation into Cancer and Nutrition (EPIC)-Potsdam cohort (27,548 adults) $[5,6]$. Higher levels of serum glycine and C18:2 LPC were associated with a decreased risk of T2D whereas many metabolites of diacylphosphatidylcholines among other metabolites were associated with a higher risk of T2D; these metabolites significantly improved T2D prediction compared with established risk factors and its alterations were associated early with a higher risk of T2D [6].
Interestingly on the same path, Menni and colleagues have searched for novel molecular markers for T2D and impaired fasting glucose (IFG) in a large population-based cohort of 2,204 females and they've used a nontargeted metabolomics approach of 447 fasting plasma metabolites. They've confirmed that fourteen metabolites were found to be associated with IFG. Among the identified metabolites, the branched-chain keto-acid metabolite 3-methyl-2-oxovalerate was the strongest predictive biomarker for IFG after glucose. The association was replicated in an independent population $(n=720)$ and validated in 189 twins and the research team concluded that the test may be one of the potential novel targets for clinical prediction [7].

Moreover, Padberg and colleagues have revealed that a metabolic signature including glyoxylate was associated with T2D as well as pre-diabetic population, independent of the fasting status and of occurrence of another major disease. Glyoxylate was found to be significantly increased up to three years prior to diagnosis of diabetes in non-fasted subjects as well as in fasted Type 2 diabeticpatients and this research team concluded that it could represent a new marker for the detection of medical subgroups of diabetic subjects [8]. Again, high ferritin, low glycine, and altered hepatic-derived lipid concentrations in the circulation have beenrecently proven to be associated with total red meat consumption and, independent of red meat, with diabetes risk [9].

Animal studies were also very fruitful; in 2007 , in a multi species study, Salek and colleagues have demonstrated profound changes in the urinary levels of nucleotide metabolism, including that of $\mathrm{N}$-methylnicotinamide and $\mathrm{N}$-methyl-2-pyridone-5-carboxamide, and suggested that this may provide unique biomarkers for following type 2 diabetes mellitus progression [10]. Recently, two distinct urinary

Correspondence to: Mina T Kelleni, Department of Pharmacology, Faculty of Medicine, Minia University, Egypt, Tel: (+20) 1200382422; E-mail: drthabetpharm@yahoo.com

Key words: Type 2 diabetes, metabolomics, biomarkers glycine and choline

Received: June 04, 2015; Accepted: June 28, 2015; Published: July 01, 2015 
metabolites (2-phenylethanol glucuronide, 2-(4-hydroxy-3-methoxyphenyl)acetaldehyde sulfate) have been also identified in male Wistar rats and proved to be involved in T2D development [2].

Finally, Cobb and colleagues have recently suggested that some metabolites including glycine but specifically, a-ketobutyric acid and linoleoylglycerophosphocholine rival fasting blood glucose in their ability to predict IGT as well as a number of metabolites that are complementary with and additive to FPG when utilized in multivariate models for the prediction of IGT. They've introduced a novel all metabolite IGT test that includes a diverse set of metabolites representing a diverse set of biochemical pathways: a ketone body, a fatty acid, a branched-chain amino acid catabolite, an essential vitamin, an amino acid, a lysophosphocholine, and a shunt metabolite from $\alpha$-ketobutyric acid ( $\alpha-\mathrm{HB})$. They've suggested that this test that requires only a single fasted blood draw is more convenient than the oral glucose tolerance test [11]. I really think they're walking in the right path and I strongly recommend that several other global teams should join and contribute to develop easier and more specific tests.

\section{References}

1. Zhang AH, Qiu S, Xu HY, Sun H, Wang XJ (2014) Metabolomics in diabetes. Clin Chim Acta 429: 106-110. [Crossref]

2. Sun H, Zhang S, Zhang A, Yan G, Wu X, et al. (2014) Metabolomic analysis of dietinduced type 2 diabetes using UPLC/MS integrated with pattern recognition approach. PLoS One 9: e93384. [Crossref]
3. Bain JR, Stevens RD, Wenner BR, Ilkayeva O, Muoio DM, et al. (2009) Metabolomics applied to diabetes research: moving from information to knowledge. Diabetes 58: 2429-2443. [Crossref]

4. Park S, Sadanala KC, et al. (2015) A Metabolomic Approach to Understanding the Metabolic Link between Obesity and Diabetes. Mol Cells. [Crossref]

5. Wang-Sattler R, Yu Z, Herder C, Messias AC, Floegel A, et al. (2012) Novel biomarkers for pre-diabetes identified by metabolomics. Mol Syst Biol 8: 615. [Crossref]

6. Floegel A, Stefan N, Yu Z, Mühlenbruch K, Drogan D, et al. (2013) Identification of serum metabolites associated with risk of type 2 diabetes using a targeted metabolomic approach. Diabetes 62: 639-648. [Crossref]

7. Menni C, Fauman E, Erte I, Perry JR, Kastenmüller G, et al. (2013) Biomarkers for type 2 diabetes and impaired fasting glucose using a nontargeted metabolomics approach. Diabetes 62: 4270-4276. [Crossref]

8. Padberg I, Peter E, González-Maldonado S, Witt H, Mueller M, et al. (2014) A new metabolomic signature in type-2 diabetes mellitus and its pathophysiology. PLoS One 9: e85082. [Crossref]

9. Wittenbecher C, Mühlenbruch K, Kröger J, Jacobs S, Kuxhaus O, et al. (2015) Amino acids, lipid metabolites, and ferritin as potential mediators linking red meat consumption to type 2 diabetes. Am J Clin Nutr 101: 1241-1250. [Crossref]

10. Salek RM, Maguire ML, Bentley E, Rubtsov DV, Hough T, et al. (2007) A metabolomic comparison of urinary changes in type 2 diabetes in mouse, rat, and human. Physiol Genomics 29: 99-108. [Crossref]

11. Cobb J, Eckhart A, Perichon R, Wulff J, Mitchell M, et al. (2015) A novel test for IGT utilizing metabolite markers of glucose tolerance. J Diabetes Sci Technol 9: 69-76. [Crossref]

Copyright: (C)2015 Kelleni MT. This is an open-access article distributed under the terms of the Creative Commons Attribution License, which permits unrestricted use, distribution, and reproduction in any medium, provided the original author and source are credited. 